\title{
Nonlinear Parameters for Monitoring Gear: Comparison Between Lempel-Ziv, Approximate Entropy, and Sample Entropy Complexity
}

\author{
Mourad Kedadouche, Marc Thomas, Antoine Tahan, and Raynald Guilbault \\ Department of Mechanical Engineering, École de Technologie supérieure, 1100 Notre-Dame Street West, \\ Montreal, QC, Canada H3C 1 K3 \\ Correspondence should be addressed to Marc Thomas; marc.thomas@etsmtl.ca
}

Received 15 September 2014; Revised 16 March 2015; Accepted 14 April 2015

Academic Editor: Miguel Neves

Copyright (C) 2015 Mourad Kedadouche et al. This is an open access article distributed under the Creative Commons Attribution License, which permits unrestricted use, distribution, and reproduction in any medium, provided the original work is properly cited.

\begin{abstract}
Vibration analysis is the most used technique for defect monitoring failures of industrial gearboxes. Detection and diagnosis of gear defects are thus crucial to avoid catastrophic failures. It is therefore important to detect early fault symptoms. This paper introduces signal processing methods based on approximate entropy (ApEn), sample entropy (SampEn), and Lempel-Ziv Complexity (LZC) for detection of gears defects. These methods are based on statistical measurements exploring the regularity of vibratory signals. Applied to gear signals, the parameter selection of ApEn, SampEn, and LZC calculation is first numerically investigated, and appropriate parameters are suggested. Finally, an experimental study is presented to investigate the effectiveness of these indicators and a comparative study with traditional time domain indicators is presented. The results demonstrate that ApEn, SampEn, and LZC provide alternative features for signal processing. A new methodology is presented combining both Kurtosis and LZC for early detection of faults. The results show that this proposed method may be used as an effective tool for early detection of gear faults.
\end{abstract}

\section{Introduction}

Gearboxes play an important role in industrial applications and unexpected failures often result in significant economic losses. Numerous papers considering gear condition monitoring through vibration measurements were published over the years. Compared to classical techniques such as statistical time indicators or Fast Fourier Transform, advanced signal processing techniques like time-frequency analysis (STFT, Wigner-Ville) [1-4] or wavelet transform $[5,6]$ have shown to be more efficient for gear defect detection. Baydar et al. [7-10] proposed various methods such as the instantaneous power spectrum, Wigner-Ville distribution, and the wavelet transform method for local tooth fault detection from vibration and acoustic signals. Yesilyurt [11] applied the spectrogram and scalogram approach for gearbox fault detection.

Various researches are focused on the understanding of dynamic gears behaviour. Parey and Tandon [12] give a review of the dynamic modelling of spur gears including defects. The effects of various nonlinearities, such as variable mesh stiffness, mesh damping, gear errors, effect of friction, and backlash, on gear dynamic behaviour have been discussed. Due to instantaneous variations of these effects, gear systems are often characterised by nonlinear behaviours.

Randall [13] classified the vibrations generated by gear meshing as being caused by deviations from the ideal tooth profile producing a vibration signal at the tooth-meshing frequency. These variations in tooth loading generate amplitude modulations and fluctuations in the rotational speed. Nonuniform tooth spacing produces frequency modulation effects and local tooth faults generate additional impulses. Imperfections (errors) in the gear during manufacture will also be a source of vibration. The instantaneous variations of the effects cited above may be considered as the first steps of degradation of the gear. At this stage, a modification of the characteristics of dynamics responses may be observed. 
However, in the early stages of fault development, main frequencies have low amplitudes and can be masked by other vibration sources or buried in background noise.

A large number of statistical metrics have been applied for monitoring gear defects, such as the root mean square, Crest Factor, Kurtosis, FM0, FM4, FM4*, M6, M6A, M6A*, NA4, NA4* ${ }^{*}, \mathrm{NB} 4, \mathrm{NB} 4^{*} \ldots$ [14-16]. Most of these measures characterize the statistical properties of vibration and give a global view to provide any recognition of localized phenomena. Stevens et al. [17] noted that these measures are suitable for detection and diagnosis when mechanical faults take the form of impulses which impose periodic pulses of short time duration. However, Feng et al. [18] noted that these parameters cannot give a perception into the gear dynamic system generating the vibration signals. Wu et al. [19] used the standard deviation for monitoring tooth cracks of gear, but it has been proven to be ineffective for tooth crack detection.

Therefore, it is interesting to use others techniques as nonlinear estimations of parameters which may be a good alternative to monitoring hidden faults in the measured signals. Yan and Gao [20] give a review of a large number of nonlinear parameter identification techniques. Wang et al. [21] researched the application of some nonlinearity analysis methods, including the pseudophase portrait, singular spectrum, and correlation dimension, for monitoring and diagnosis of rotating machinery. Jiang et al. [22] used the correlation dimension for monitoring gearbox. Feng et al. [18] investigated the irregularity of the gear signal using the regularisation dimensions.

As said before, the effects of various nonlinearities cause a variation in the dynamic behaviour of the gear. As the default develops, more contact pressure between two mating parts changes. The contact pressure variation results in amplitude and frequency modulations and hence more frequency will appear in the frequency domain with their harmonics and an increase of the noise can be observed. Therefore, the fault progress leads to complexity (randomness) changes because of a variation of the contact pressure between two mating parts. The complexity of a signal increases when more frequency components exist. On the other hand, when the defect increases, we observe more frequency components in the signal and we can use the complexity of the signal as a quantitative measurement to evaluate the severity of the defect. The complexity of the signal can be described by the approximate entropy (ApEn), the sample entropy (SampEn), and the Lempel-Ziv complexity (LZC). These parameters are nonlinear parameters which can be used to characterise the regularity of the signal.

The Lempel-Ziv complexity (LZC), sample entropy (Sam$\mathrm{pEn}$ ), and approximate entropy (ApEn) thus present alternative tools for signal analysis involving nonlinear dynamics. These methods are becoming more popular and have found wide applications in various disciplines, especially in the field of biomedical engineering. ApEn has recently received more attention. Yan and Gao [20] investigated the application of ApEn for the health monitoring of rolling element bearings. He and Zhang [23] applied the ApEn for monitoring signal of the acoustic emission from defects in rolling element Bearings. Fu et al. [24] used the ApEn method to fault signal analysis in electric power system. Using ApEn, Xu et al. [25] detected the looseness of the bearing bushing in turbo generator. In all these work, ApEn is used as a nonlinear feature parameter for analysing the vibration signal and effectively identifying the conditions of the mechanical system.

On the other hand, Yan and Gao [26] investigated the application of Lempel-Ziv complexity (LZC) for the health monitoring of rolling element bearings. Wang et al. [27] compare and analyse quantitative diagnosis methods based on Lempel-Ziv complexity for bearing faults, using continuous wavelet transform (CWT), Empirical Mode Decomposition (EMD) method, and wavelet packet method for decomposition of vibration signal. Kedadouche et al. [28] combined LZC and EMD for early detection of gears cracks.

The use of SampEn was introduced by Dennis Wong et al. [29] which was the improvement of a previous method, namely, approximate entropy (ApEn). However, sample entropy was not often used in monitoring of rotating machine. Dennis Wong et al. [29] used sample entropy for classification of ball bearing faults. Zhang et al. [30] used multiscale entropy and adaptive neurofuzzy inference for bearing fault classification. The sample entropy was discussed and implemented in many areas such as physiological time series analysis [31], heart rate variability [32], and neural respiratory signal [33]. Sun et al. [34] proposed a health auxiliary method based on SampEn for lead-acid battery unit. Widodo et al. [35] proposed intelligent prognostics for battery health based on sample entropy (SampEn) features of discharge voltage. The SampEn feature can depict degradation condition of battery and represents the health condition of battery.

As illustrated above, LZC, SampEn, and ApEn are becoming more and more attractive in the field of detection and fault diagnosis. However, no work has been found to apply ApEn, LZC, or SampEn measurement for diagnosis of gear faults. Therefore, in this paper, ApEn, SampEn, and LZC are introduced to analyse vibration signals from gear and investigate their efficiency for the defect detection and severity evaluation of gears faults.

\section{Theoretical Background}

2.1. Approximate Entropy. Consider a time series $S(i), i=$ $0, \ldots, N$. Its "regularity" may be measured by ApEn in a multiple dimensional space so that series of vectors are constructed and expressed as follows:

$$
\begin{aligned}
X & (N-m+1) \\
& =\{x(N-m+1), x(N-m+2), \ldots, x(N)\} .
\end{aligned}
$$

Each vector is composed of $m$ consecutive and discrete point data of the time series $S$. The distance between two vectors $X(i)$ and $X(j)$ can be defined as the maximum difference between their respective corresponding elements:

$$
\begin{aligned}
d & (X(i), X(j)) \\
& =\max _{k=1,2, \ldots, m}(|x(i+k-1)-x(j+k-1)|),
\end{aligned}
$$


where $i=1,2, \ldots, N-m+1, j=1,2 \ldots, N-m+1$, and $N$ is the number of data points contained in the times series.

For each vector $X(i)$, a measurement that describes the similarity between the vectors $X(i)$ and all other vectors $X(j)$ can be defined as

$$
C_{i}^{m}(r)=\frac{1}{N-(m-1)} \sum_{j \neq i} \Theta\{r-d(X(i), X(j))\},
$$

where

$$
\Theta\{x\}= \begin{cases}1, & x \geq 0 \\ 0, & x<0 .\end{cases}
$$

The symbol $r$ represents a predetermined tolerance value. $r=$ $k \times s d_{S}$, where $k$ is a positive constant $(k>0)$ and $\operatorname{std}_{S}$ is the standard deviation of the time series $S$.

A finite time series consisting of $N$ data points is used to estimate the ApEn value of the time series, which is defined as

$$
\begin{aligned}
\operatorname{ApEn}(m, r, N) & =\emptyset^{m}(r)-\emptyset^{m+1}(r), \\
\emptyset^{m}(r) & =\frac{1}{N-m+1} \sum_{i=1}^{N-m+1} \ln \left[C_{i}^{m}(r)\right] .
\end{aligned}
$$

2.2. Sample Entropy. Sample entropy was developed because it has better representation of the entropy in the analysed signals as comparing with the original approximate entropy (ApEn) [31]. The motivation for this method is the classification of complex systems that includes both deterministic and stochastic features from time series with a limited number of data points [32]. Higher values of sample entropy describe more irregularities in the time series.

For a given $N$ total number of data points, we form the $N-m+1$ vectors $X_{m}(i)$ as

$$
\begin{aligned}
X_{m}(i)=\{x(i), x(i+1), \ldots, x(i+m-1)\}, & \\
i & =1 \text { to } N-m+1,
\end{aligned}
$$

where $m$ is the length of sequences to be compared.

The distance between such vectors is defined as

$$
\begin{array}{ll}
d_{m} & \left(X_{m}(i), X_{m}(j)\right) \\
\quad=\max \left(\left|X_{m}(i+k)-X_{m}(j+k)\right|\right), & \\
& 0 \leq k \leq m-1 .
\end{array}
$$

For $i=1$ to $N-m+1$, let:

$$
B_{i}^{m}(r)=\frac{1}{N-m+1} v^{m}(i),
$$

where $r$ is the tolerance for accepting matrices and $v^{m}$ is the number of $d_{m}\left(X_{m}(i), X_{m}(j)\right)$, for $i \neq j$.

Similarly, we define another function:

$$
A_{i}^{m}(r)=\frac{1}{N-m+1} w^{m+1}(i)
$$

where $w^{m+1}$ is the number of $d_{m+1}\left(X_{m+1}(i), X_{m+1}(j)\right) \leq r$, for $i \neq j$.

The symbol $r$ represents a predetermined tolerance value, where $k$ is a positive constant $(k>0)$ and $s \mathrm{~d}_{S}$ is the standard deviation of the time series:

$$
r=k \times \operatorname{std}_{S}
$$

We can determine the expression of probability matching points:

$$
\begin{aligned}
& B^{m}(r)=\frac{1}{N-m+1} \sum_{i=1}^{N-m} B_{i}^{m}(r), \\
& A^{m}(r)=\frac{1}{N-m+1} \sum_{i=1}^{N-m} A_{i}^{m}(r),
\end{aligned}
$$

where $B^{m}(r)$ represents the probability that two sequences will match for $m$ points, whereas $A^{m}(r)$ is the probability that two sequences will match for $m+1$ points. The sample entropy is then defined as

$$
\text { Sample Entropy }=-\ln \left[\frac{A^{m}(r)}{B^{m}(r)}\right]
$$

2.3. Complexity Analysis. The complexity analysis is based on the Lempel-Ziv definition [36]. This approach transforms the analysed signal into a data sequence. To illustrate the procedure, consider a gear vibration signal with a known mean value. A new sequence $(S)$ is reconstructed by comparing the value of each sample of the previous sequence within the mean value. If the value of the sample is larger, it is set to one (1), otherwise to zero (0). Therefore, only two binary symbols are present in the new data sequence. This $S$ is subsequently scanned from its first sample to its end. When a subsequence that is not encountered in the previous scanning process is discovered, the complexity value is increased by one (1). Thus, the Lempel-Ziv complexity reflects the number of all different subsequences contained in the original sequence. Figure 1 (reproduced from [26]) described the algorithm.

For generality sake, normalized complexity $C(n)$ is often used to obtain a measure independent of the sequence length:

$$
\begin{aligned}
& C(n)=\frac{c(n)}{b(n)}, \\
& b(n)=\frac{N}{\log ^{2}(N)} .
\end{aligned}
$$

\subsection{Parameters Selection of ApEn, SampEn, and LZC for Gear} Signals. From the above described algorithm of ApEn and SampEn, it can be seen that the calculated ApEn and SampEn values depend on two parameters which are the embedded dimension $m$ and the tolerance $r$.

However, no guidelines exist for optimizing their values. In order to simulate the vibratory signals of gearbox, a gear multiplicative model whose meshing is modulated in 


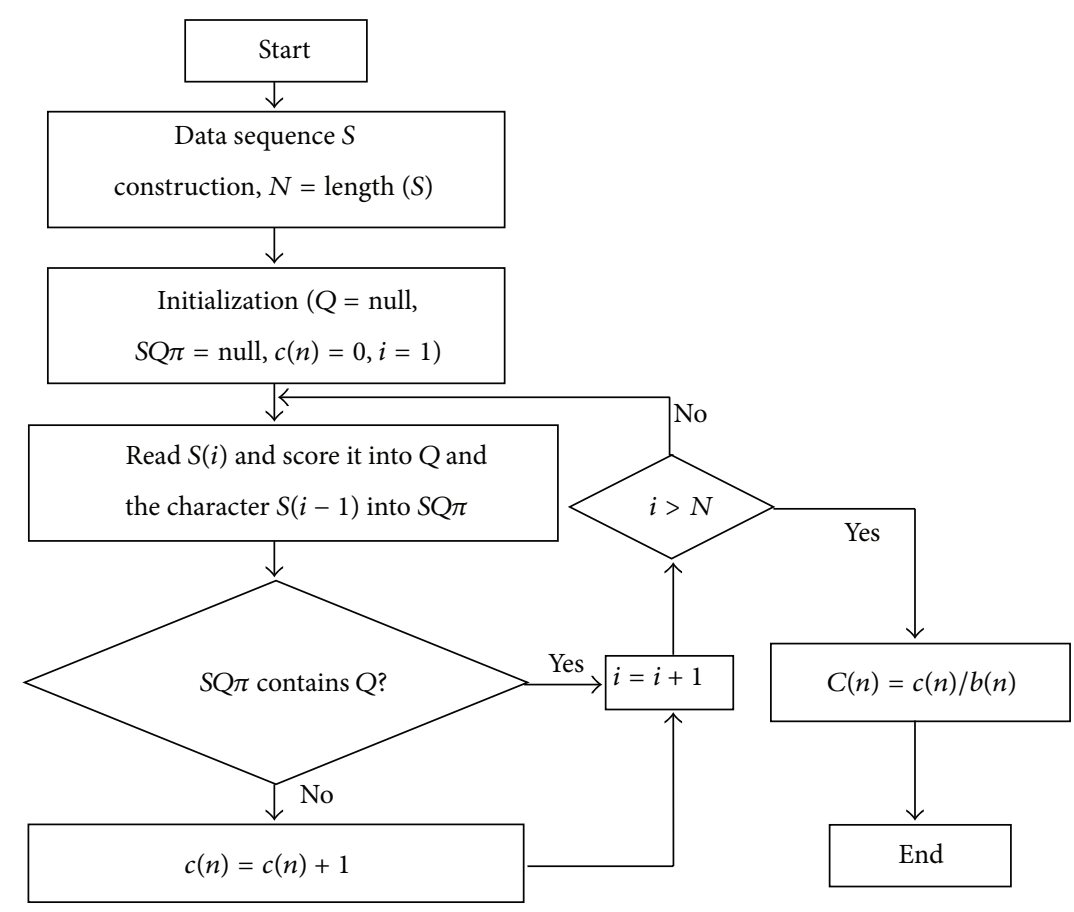

FIGURE 1: The flow chart of LZC algorithm.

TABLE 1: Geared system data of the simulated signal.

\begin{tabular}{lcc}
\hline & Frequency $(\mathrm{Hz})$ & Amplitude \\
\hline Pinion: number of teeth $=20$ & 15 & 0.15 \\
Gear: number of teeth $=21$ & 14.28 & 0.15 \\
Gear mesh: the first harmonic & 300 & 1 \\
Gear mesh: the second harmonic & 600 & 0.6 \\
Gear mesh: the third harmonic & 900 & 0.3 \\
\hline
\end{tabular}

amplitude has been used. The gear model as defined in [37] is used:

$$
\begin{aligned}
x(t)= & \sum_{m=-\infty}^{+\infty} S_{r 1}\left(t-m \tau_{r 1}\right)+\sum_{m=-\infty}^{+\infty} S_{r 2}\left(t-m \tau_{r 2}\right) \\
& +\sum_{n=-\infty}^{+\infty} S_{e}\left(t-n \tau_{e}\right) \\
& \cdot\left(1+\sum_{m=-\infty}^{+\infty} S_{r 1}\left(t-m \tau_{r 1}\right)\right),
\end{aligned}
$$

where $\tau_{e}, \tau_{r 1}$, and $\tau_{r 2}$ represent the meshing period and the rotational periods. $S_{e}(t), S_{r 1}(t)$, and $S_{r 2}(t)$ represent the meshing signal and its modulation.

Table 1 gives the parameters simulation of the model described in (14).

Figures 2 and 3 represent the simulated signal and its spectrum, respectively.

For a given dimension $m$, the key step of the ApEn algorithm is to calculate the distance between two vectors and to count the number of distances less than $r$ (where $r=k^{*}$ standard deviation of the time series), as discussed in [20].

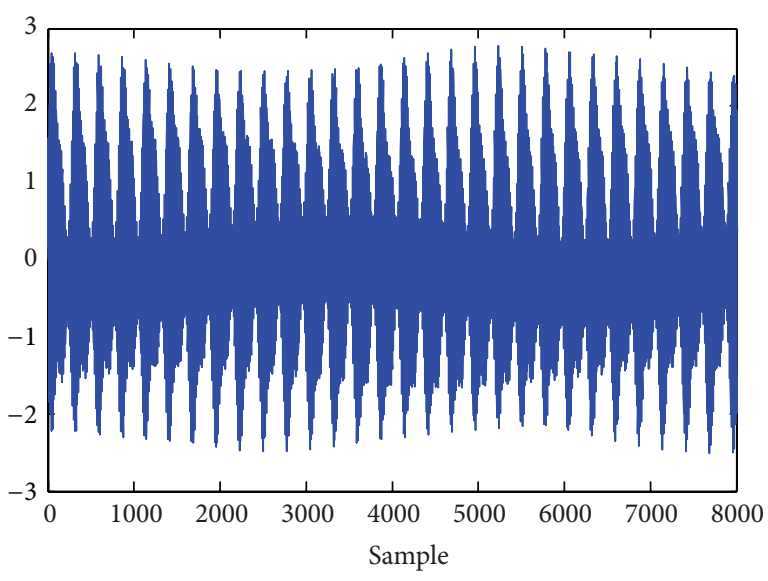

Figure 2: The simulated signal.

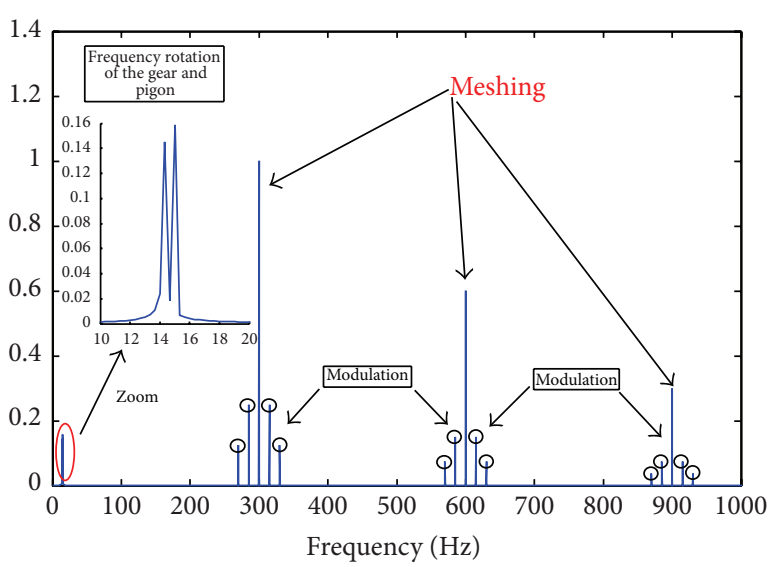

FIgURE 3: Spectrum of the simulated signal. 


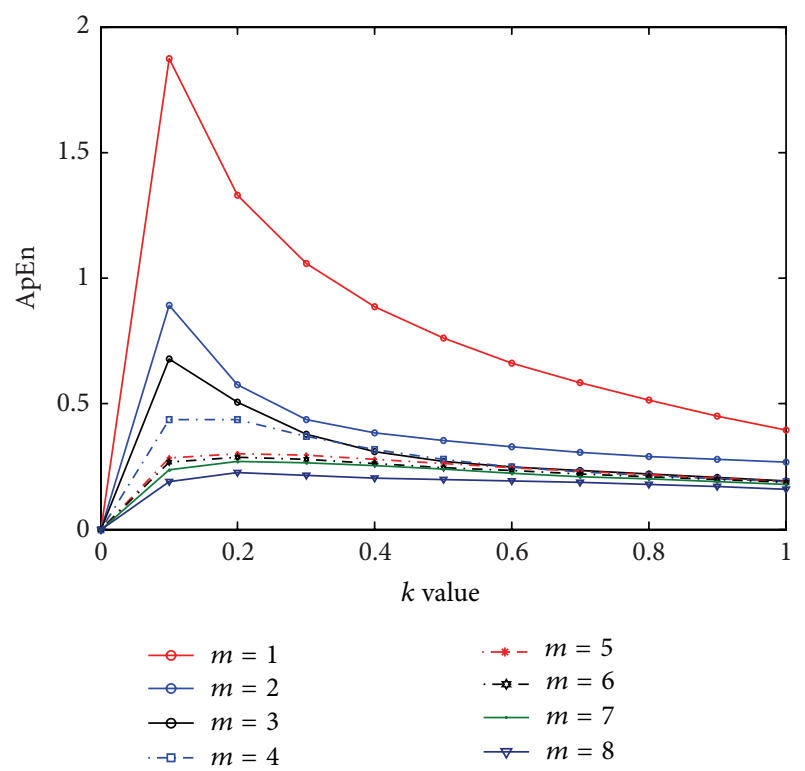

Figure 4: The calculated ApEn values by different parameters $(k$ value and $m$ ).

The selections of $k$ and $m$ are based on a convergence analysis [20, 23-26]. The choice of $m$ and $k$ depends on the nature of the time series treated. The best parameters to be chosen are those who present little fluctuation or on the other hand a good reproducibility.

From Figure 4, it can be seen that when $m=1$, ApEn has the poorest convergence property (a big fluctuation). With $m$ increasing (more than 2), the convergence property of ApEn becomes better for $k$ greater than 0.4 .

Like ApEn, sample entropy (SampEn) depends on the two parameters $m$ and $k$. Therefore, the determination of these two parameters is critical. The same procedure was undertaken for the selection of $k$ and $m$. The same characteristic was obtained as ApEn. From Figure 5 we can see that, for $m=2$, the SampEn presents a good convergence when $k$ is greater than 0.5 .

However, a larger $m$ will lead to much higher computational cost. Actually, when $m=2$, the convergence property of ApEn and SampEn is already good enough. Of course, the $k$ value should also be a compromise to avoid losing much detailed system information.

By this investigation, $m=2$ and $k$ value $=0.5$ times the standard deviation are selected for ApEn and SampEn calculation of the vibration signal in this paper.

The relationship between the ApEn, SampEn, and LZC values and the data length are illustrated in Figure 6. In Figures 7 and 8, seven simulated signals are comparatively displayed under sampling rates of $4,8,12,16,20$, and $24 \mathrm{kHz}$, respectively. It is seen, in three cases (ApEn, SampEn, and LZC), that when the data length is greater than 5000 points, the variation of ApEn, SampEn, and LZC with respect to each frequency sampling rate becomes insignificant.

However, the sampling frequency and the data length are linked. So we must take consideration of the component

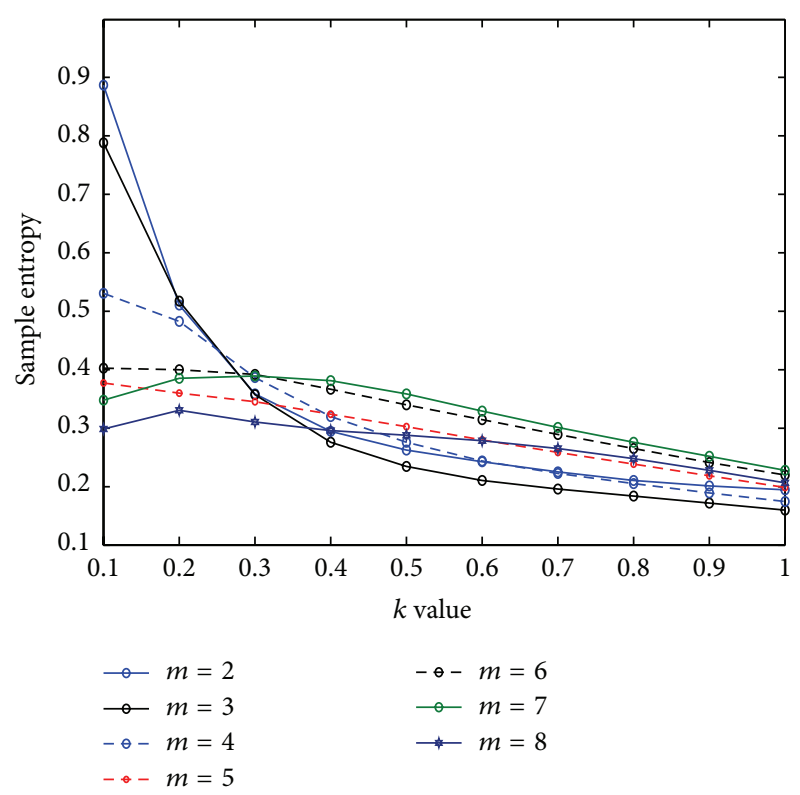

FIGURE 5: The calculated sample entropy values by different parameters $(k$ value and $m)$.

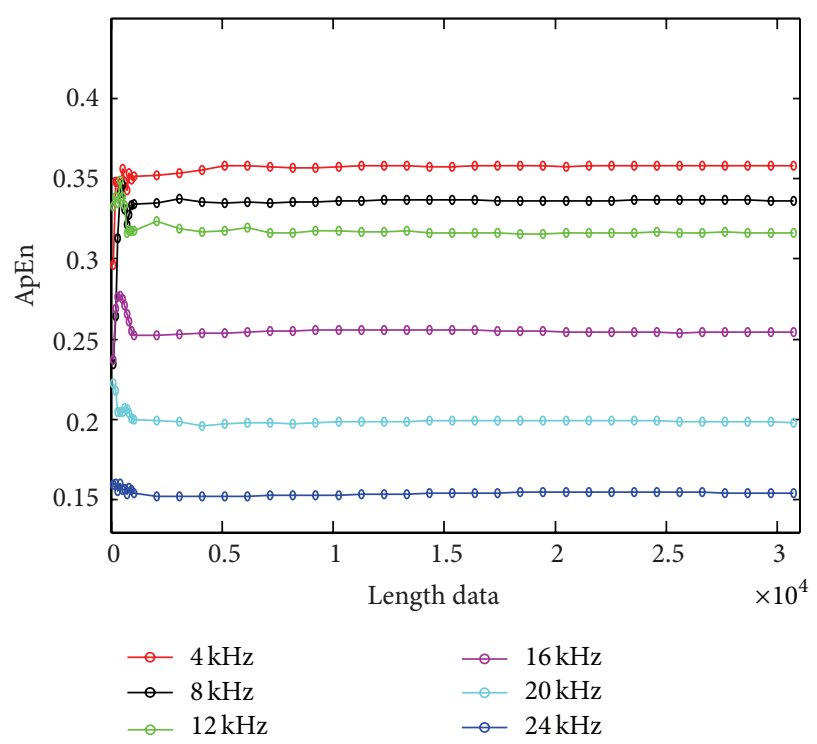

FIGURE 6: The calculated ApEn values by different parameters (length of the data and the sample frequency).

defining the signal. The sample frequency must be more than 2 times the highest frequency presented in the signal, for Shannon respect. In our case, the sample frequency must be 2 times greater than the third meshing frequency $(900 \mathrm{~Hz})$. The simulation was conducted with $F_{s}$ greater than $4 \mathrm{kHz}$ which is sufficient enough. On the other hand, for a better detection of the modulations, we must choose a good resolution frequency which is defined by $\Delta H=F_{s} /$ length(signal). For the simulated signal, the modulations are about $\pm 15 \mathrm{~Hz}$. So, a frequency resolution of $1 \mathrm{~Hz}$ is enough. This means that the 


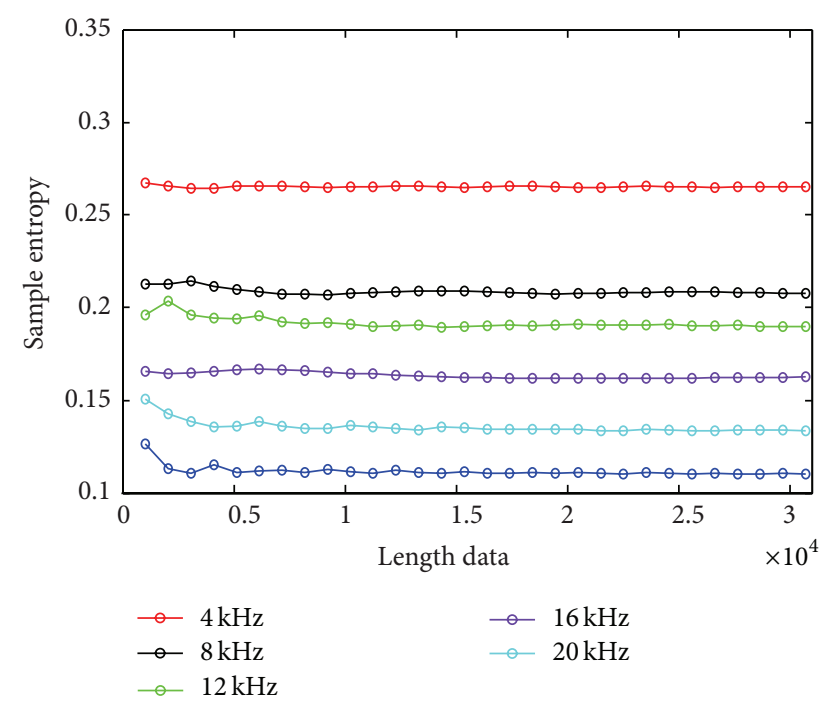

FIgURE 7: The calculated SampEn values by different parameters (length of the data and the sample frequency).

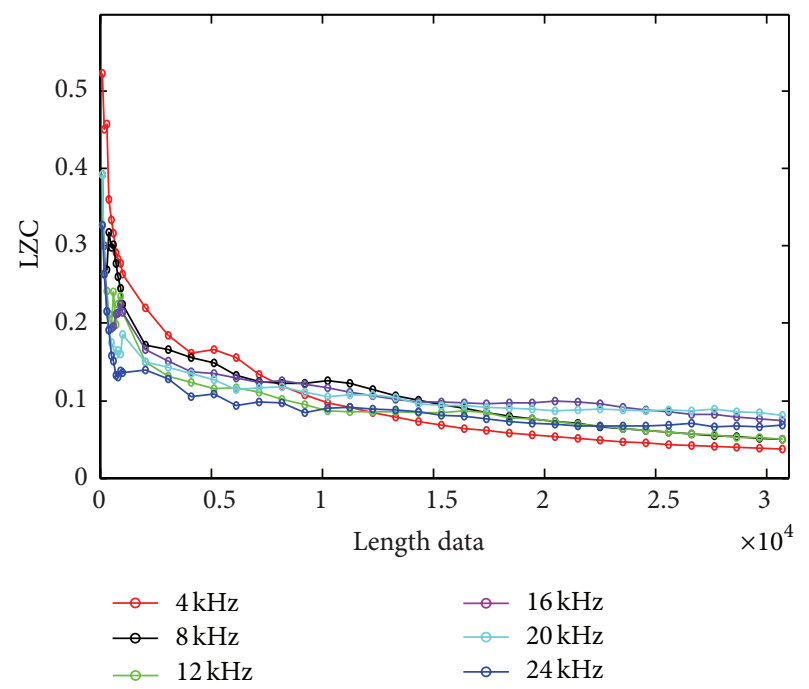

FIGURE 8: The calculated LZC values by different parameters (length of the data and the sample frequency).

minimum length of data to be analysed is equal to the sample frequency $(4000 \mathrm{~Hz})$.

Figure 9 shows a comparison of computing time between LZC, ApEn, and SampEn.

It is clear that the LZC is the best method for time computing. The sample entropy algorithm is simpler than the ApEn algorithm, requiring approximately one-half time to calculate.

2.5. Influence of Noise. It is well known that a white noise contains most abundant frequency components compared with other kinds of signal. If a signal is contaminated by a white noise, calculated ApEn, SampEn, and LZC values will also be. Using the simulated signal, the ApEn, SampEn, and

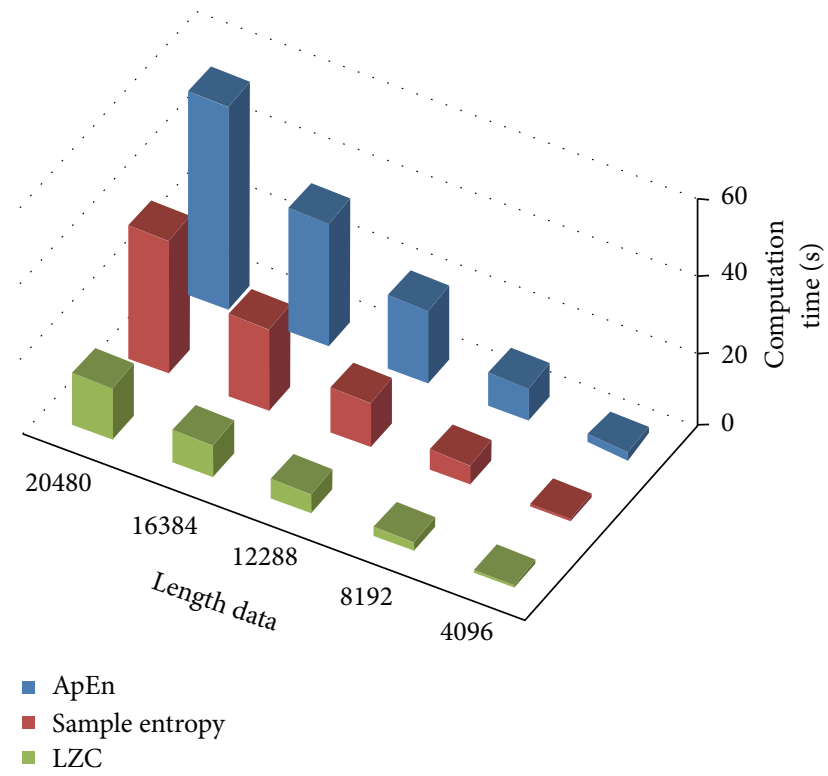

Figure 9: Time computation of both LZC and ApEn for different length of data.

TABLE 2: ApEn, SampEn, and LZC for different SNR of gear signals.

\begin{tabular}{lccc}
\hline Cases & ApEn & LZC & SampEn \\
\hline The simulated signal & 0.3529 & 0.1294 & 0.2644 \\
SNR $=80 \mathrm{~dB}$ & 0.3547 & 0.1364 & 0.2656 \\
SNR $=60 \mathrm{~dB}$ & 0.3648 & 0.1692 & 0.2765 \\
SNR $=40 \mathrm{~dB}$ & 0.4495 & 0.3056 & 0.3500 \\
SNR $=0 \mathrm{~dB}$ & 1.3163 & 0.8909 & 1.2190 \\
\hline
\end{tabular}

LZC values corresponding to different SNRs are calculated, as listed in Table 2 .

It may be noticed that the ApEn, SampEn, and LZC values increase as the SNR decreases, which corresponds to a degradation of the data quality. As discussed in [20], the working condition of machine system deteriorates due to the initiation and/or propagation of the defects. The number of frequency components contained in the signal will increase and the SNR decreases, resulting in a decrease in its regularity. This allows a decrease of its ApEn, SampEn, and LZC values. The simulation results listed in Table 2 confirm that the ApEn, SampEn, and LZC values provide a quantitative measurement for characterising a dynamic signal which can be represented by the deterioration of a machine's health condition.

From Table 2, it can be seen that ApEn and SampEn are very sensitive to large noises; however, ApEn and SampEn are nearly unaffected by the noise which stay low. Otherwise, we can say that the LZC is more sensitive to noise than ApEn and SampEn.

\section{Experimental Study}

The recordings of vibration signal were carried out at CETIM, France, on a gear system with a train of gearing, with a ratio of 20/21 functioning continuously until its destruction. 

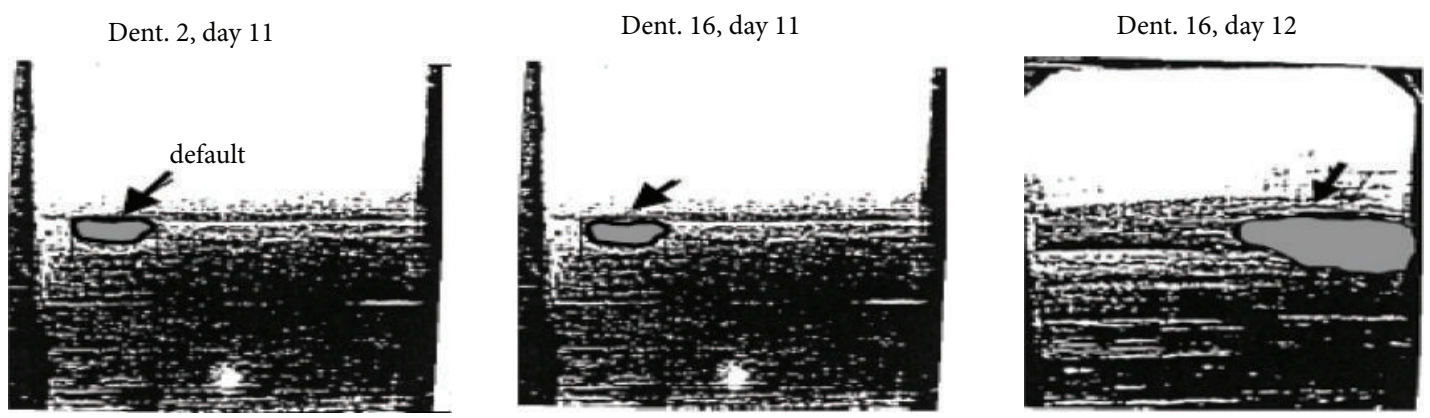

Figure 10: Evolution of the chipping.

TABLE 3: Geared system data.

\begin{tabular}{lcc}
\hline Parameter & Pinion & Gear \\
\hline Number of teeth & 20 & 21 \\
Speed (rpm) & 1000 & 952.38 \\
Drive torque (Nm) & 200 & \\
Face width (m) & 0.015 & 0.03 \\
Module (m) & 0.01 & 0.01 \\
Pressure angle & $20^{\circ}$ & $20^{\circ}$ \\
Addendum coefficient & 1.0 & 1.0 \\
Dedendum coefficient & 1.4 & 1.4 \\
\hline
\end{tabular}

TABLE 4: Daily mechanical appraisal.

\begin{tabular}{lc}
\hline Day & Observation \\
\hline 1 & No acquisition \\
2 & No anomaly \\
3 & No anomaly \\
4 & $/ / / /$ \\
5 & $/ / / /$ \\
6 & $/ / / /$ \\
7 & Chipping teeth $1 / 2$ \\
8 & No evolution \\
9 & Tooth $1 / 2:$ no evolution; tooth $15 / 16:$ start chipping \\
10 & Evolution of the chipping of the teeth $15 / 16$ \\
11 & $/ / / /$ \\
12 & // // \\
13 & Chipping across the full width of the tooth \\
\hline
\end{tabular}

The sample frequency is equal to $20 \mathrm{kHz}$. Table 3 gives the details of the gear test rig parameters. The test duration was 13 days with a daily mechanical appraisal; measurements were collected every $24 \mathrm{~h}$ except on the first day. Table 4 gives a description of the state of the gear at each $24 \mathrm{~h}$. Figure 10 presents some picture of the chipping observed in days 11 and 12. The acceleration signals for days $2,5,7,9,10$, and 12 are shown in Figure 17.

Pareya et al. [38] use the same signal for their own researches. In time domain, a multitude of indicators exists, more or less efficient for the observation of certain failures.
The most commonly used indicators are scalars from time analysis described by the equations below:

$$
\begin{aligned}
\text { RMS } & =\sqrt{\frac{1}{N} \sum_{i=1}^{N}\left(x_{i}-\bar{x}\right)^{2}}, \\
\text { Peak } & =\sup _{1 \leq i \leq N}\left|x_{i}\right|, \\
\text { CF } & =\frac{\text { Peak }}{\operatorname{RMS}}, \\
K_{\text {factor }} & =\text { Peak } \times \text { RMS, } \\
\text { Kurtosis } & =\frac{(1 / N) \sum_{i=1}^{N}\left(x_{i}-\bar{x}\right)^{4}}{\operatorname{RMS}^{4}}, \\
\text { Skewness } & =\frac{(1 / N) \sum_{i=1}^{N}\left(x_{i}-\bar{x}\right)^{3}}{\left((1 / N) \sum_{i=1}^{N}\left(x_{i}-\bar{x}\right)^{2}\right)^{3 / 2}} .
\end{aligned}
$$

The RMS, Peak, Kurtosis, Crest Factor, $K$ Factor, and Skewness of the experimental signal were considered and calculated from day 2 to day 13 (on day 1 no signal was taken).

The evolutions of these descriptors are displayed in Figures 11-16.

It can be seen that the all indicators increase greatly after day 11. After day 11 the signal becomes impulsive. The Crest Factor (CF) observes a little growth after day 9 (17\% of evolution compared to day 9).

The same observations were observed for the Peak values (26\%), the $K$ Factor (35\%), and the Skewness (20\%). This is due to the evolution of the chipping of the teeth $15 / 16$ observed in day 9. A visual chipping was observed in day 7 . The time indicators are only able to detect the deterioration after day 9.

Consequently, the classical time domains are ineffective for early detection of gear defects. These measures are appropriate for detection and diagnosis when mechanical faults are in advanced state of the degradation. As discussed in [13], at an early stage of gear degradation, the wear is preceded by a variation of different phenomena like variations in tooth loading, nonuniform tooth spacing, elastic deformation of the gear teeth, and so forth. The classical indicators cannot track these variations. For this reason, it is interesting to 


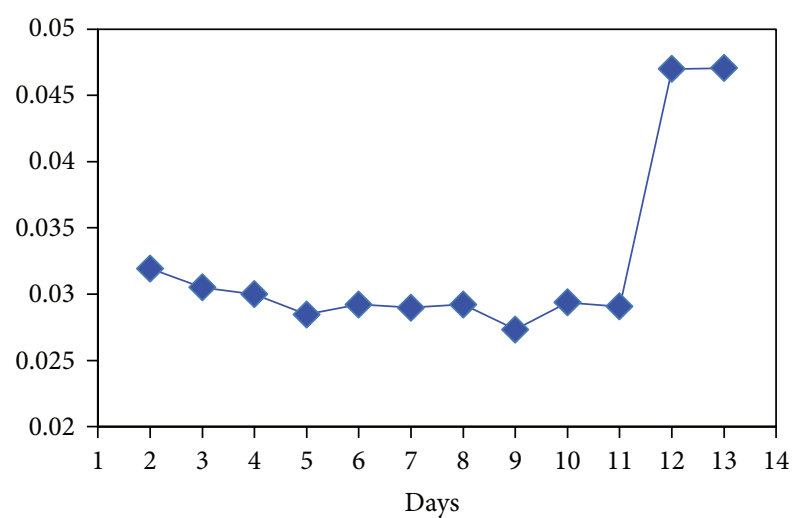

FIgURE 11: RMS $\left(\mathrm{m} / \mathrm{s}^{2}\right)$ value during the test for all days.

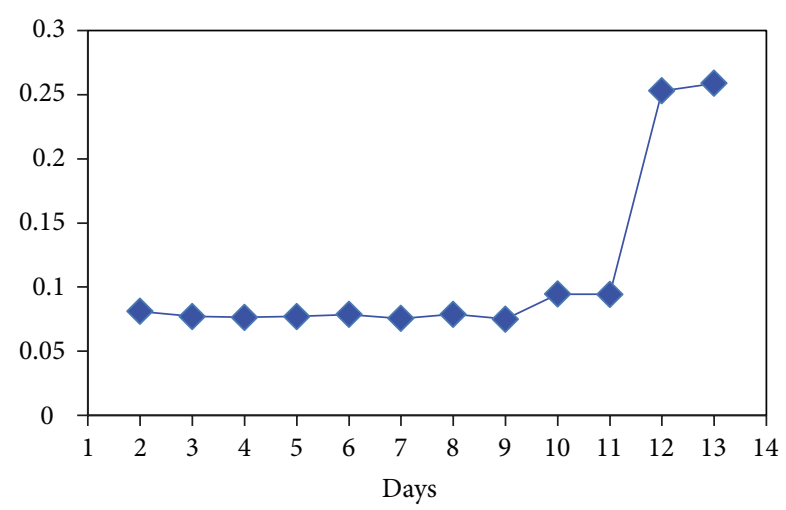

FIGURE 12: Peak $\left(\mathrm{m} / \mathrm{s}^{2}\right)$ value during the test for all days.

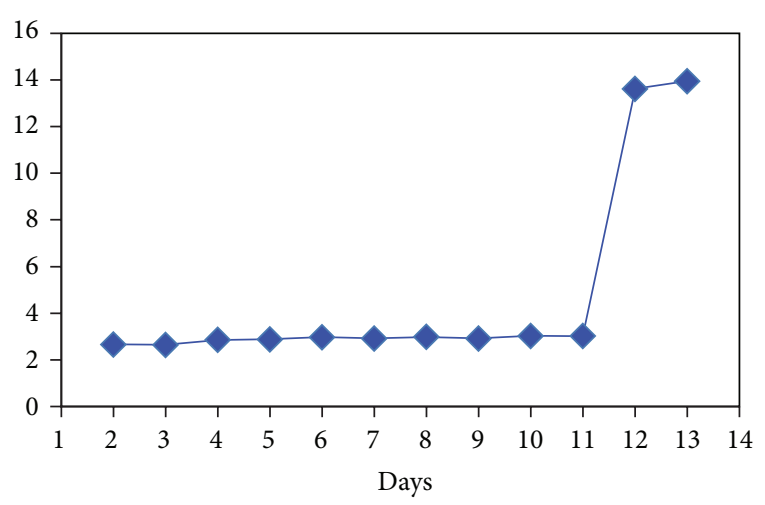

Figure 13: Kurtosis value during the test for all days.

investigate other methods which can give a perception into the gear dynamic behaviour caused by the effects cited above.

Therefore, ApEn, SampEn, and LZC of the experimental signal were computed from day 2 to day 13 (on day 1, no signal was taken). The values of ApEn and SampEn are plotted in Figure 18. The ApEn method does not present a significant increase except at day 12 . So, the characteristic ApEn seems to have equivalent performance as the classic Kurtosis. After day 11, the ApEn decreases significantly. This is due to the fact that the signal becomes impulsive and the SNR becomes higher as explained in Section 2. The SampEn

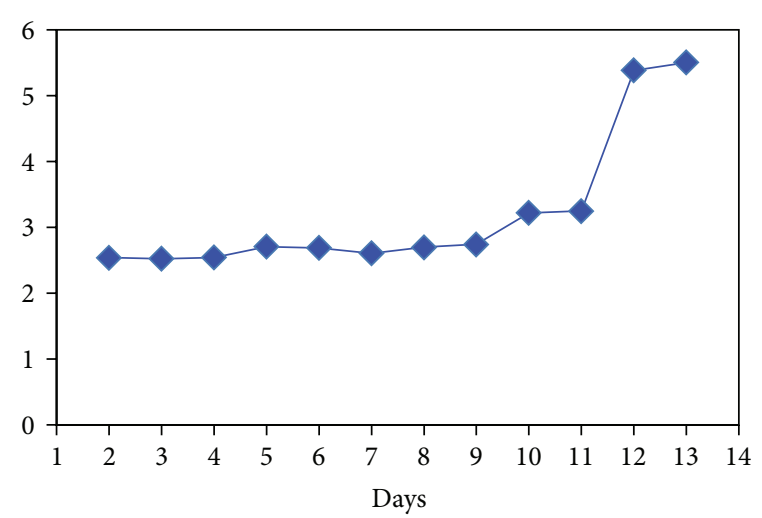

FIGURE 14: Crest Factor value during the test for all days.

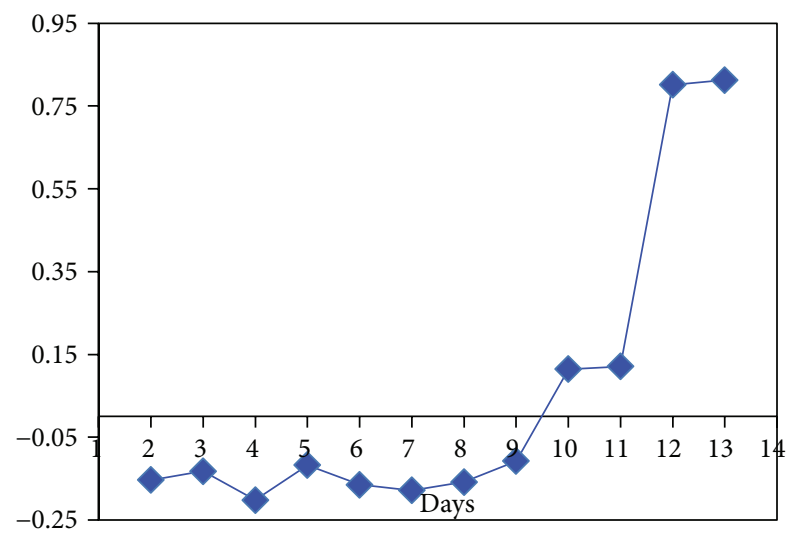

FIGURE 15: Skewness value during the test for all days.

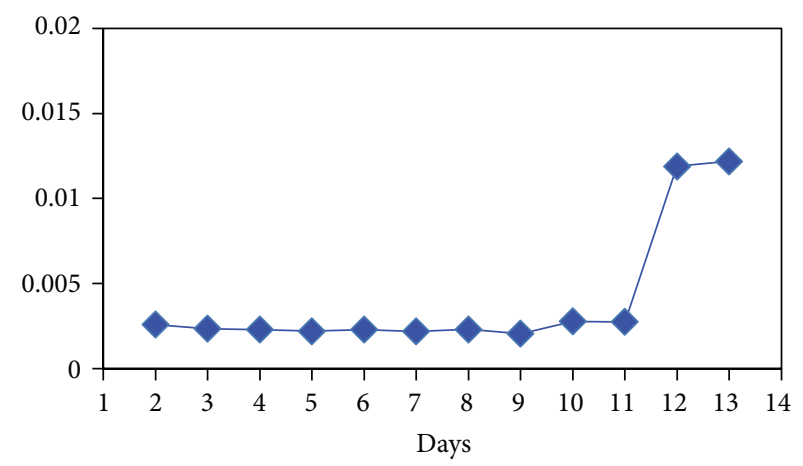

FIgURE 16: $K$ _factor value during the test for all days.

presents the same evolution as ApEn, but it presents a higher value after day 11 than ApEn. On the other hand, as explained and exhibited in Figure 9, the SampEn algorithm requires approximately one-half time to calculate than ApEn. So, it is preferable to use SampEn than ApEn. The LZC for all signals is plotted in Figure 19. The LZC shows a significant increase after day 4, revealing a clear anomaly due to gear wears. Table 4 showed that the chipping was observed clearly on day 7. That means that the beginning of the processes of the degradation was inevitably initiated before day 7. Figure 20 compares the spectrum evolution between days 4 and 5 . 

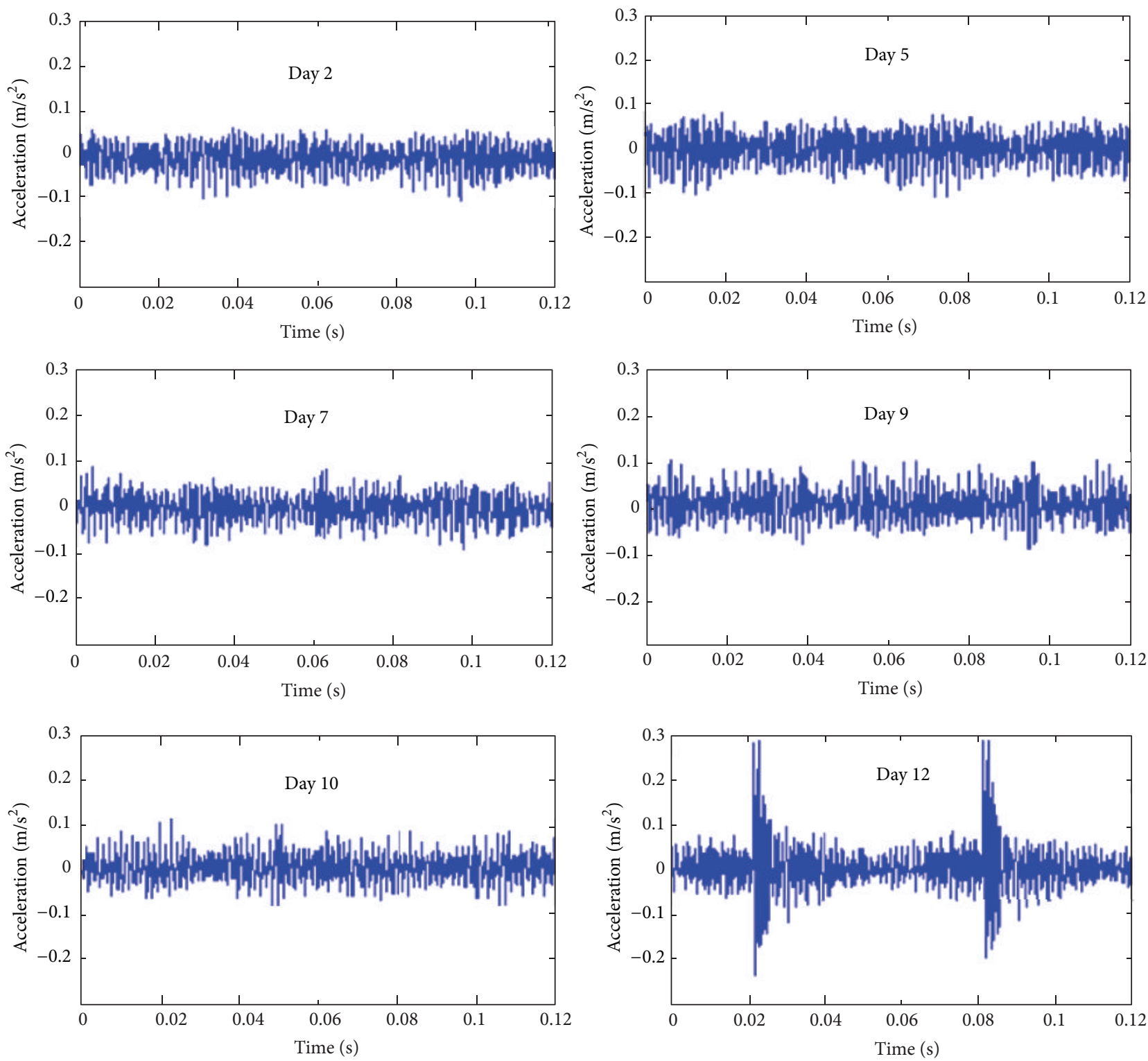

FIGURE 17: Evolution of acceleration signal with wear.

An increase of the amplitude at day 5 may be clearly noticed as compared with day 4 . Effectively, this day presents the beginning of the degradation due to wear. According to LZC measurements, the beginning of the chipping was in fact initiated at day 5 . This initiation is characterised by the growth of the frequency components related to the meshing. Consequently, these results show that LZC measurement is better than approximate entropy and sample entropy for early detection of the gear defects, in this history case.

Figure 21 presents both Kurtosis and LZC of all days. Kurtosis is reported in the abscised $X$ and LZC on $Y$. It can be seen that this plot divides the twelve days into three regions. The first region contains the three first days (day 2, day 3 , and day 4). The second encloses all days from 6 to 11. The second region is separated from the first by day 5 . Day 5 presents the change in the characteristics of the gear signal.
The last region is marked by an increase of Kurtosis and LZC stays in the same level as the second region. At this stage, the signal becomes impulsive and the gear is damaged.

Using this representation, we can easily distinguish the different steps of the process of the degradation. This representation gives both information on the impulsiveness of the signal and on the effect of the number of frequency components and noise into the signal. Consequently, this representation combining Kurtosis and LZC may be used as an efficient tool for early detection of faults.

\section{Conclusion}

This paper introduces ApEn, SampEn, and LZC metrics to analyse the vibration signal recorded from defected gears. With respect to gear signals, the parameter selection of ApEn 


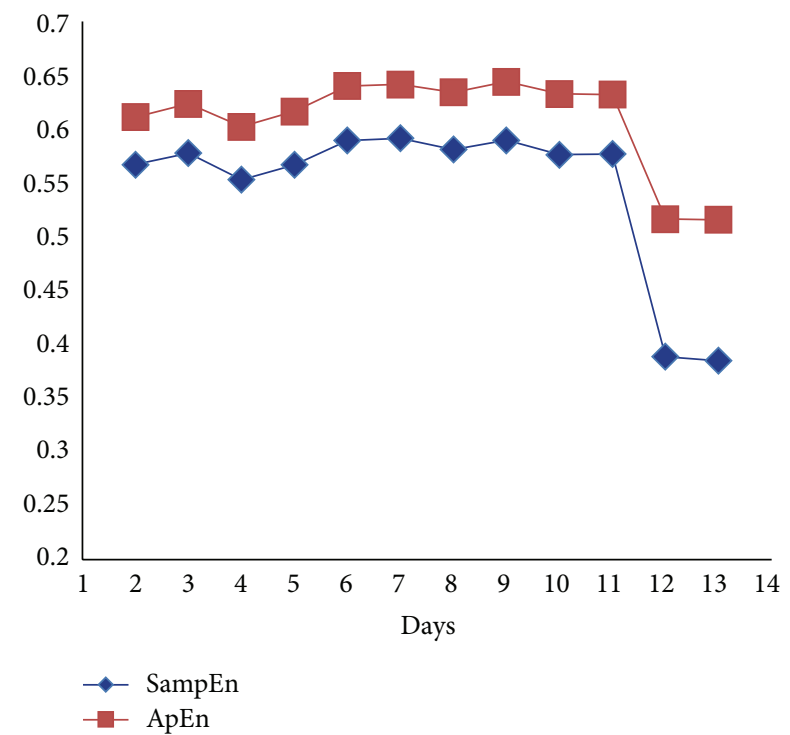

FIGURE 18: ApEn and SampEn value during the test for all days.

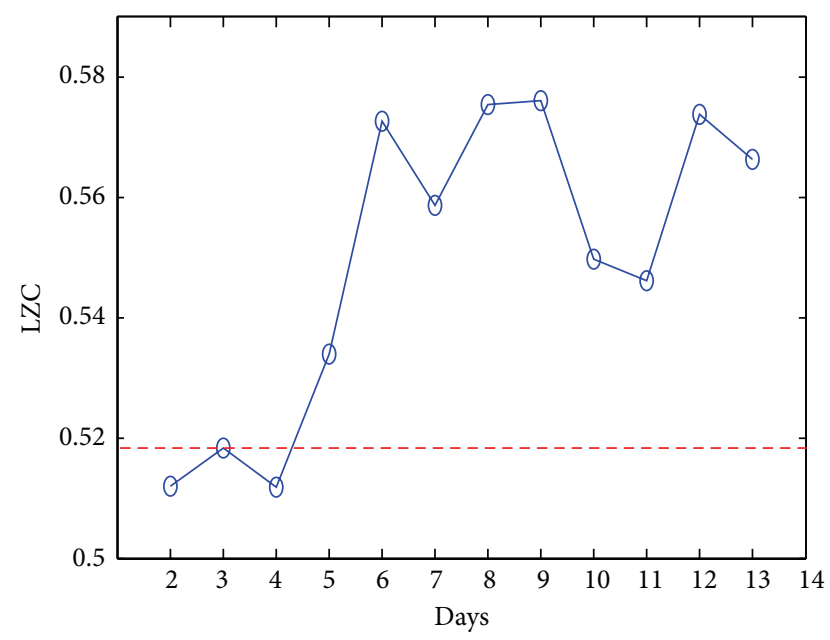

FIgURE 19: LZC value during the test for all days.

and SampEn was investigated and the results show that $m=2$ and $k$ value $=0.5$ times the standard deviation were suitable and a good compromise for the ApEn and SampEn calculation. In addition, the influence of white noise on ApEn, SampEn, and LZC calculation was also investigated. The results show that ApEn and SampEn are nearly unaffected by the noise when staying at a small level. However, ApEn and SampEn are very sensitive to the noise at high levels. LZC is more sensitive to noise as compared with ApEn. An experimental study was conducted to evaluate the effectiveness of these parameters. The results show that sample entropy is better than approximate entropy. However, LZC can detect the defect of the gears earlier than SampEn and ApEn. The classical time indicators seem to be ineffective for early detection compared to LZC. The representation in a plan (Kurtosis, LZC) is proposed as a new tool for effectively monitoring gear defects. Although experimental results look promising, the proposed vibration methodology has to be

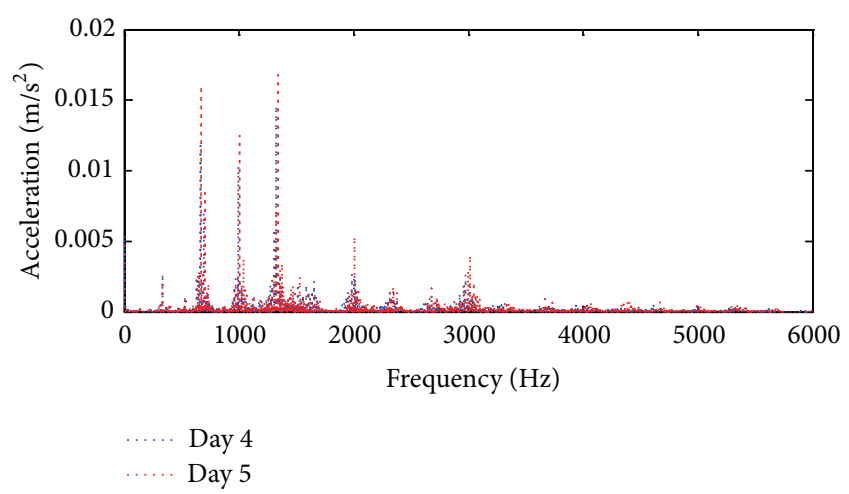

Figure 20: Spectrum of the experimental signal.

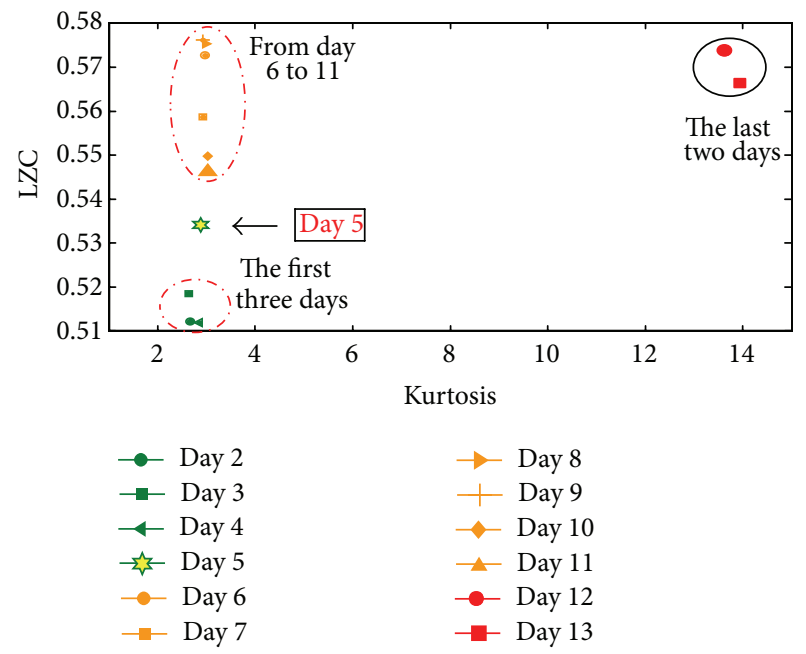

FIGURE 21: Kurtosis versus LZC value during the test for all days.

tested on the other test rig also. This research is thus being continued to analyze vibration signals from different defect types and on different types of gears, to systematically validate the efficiency of this technique.

\section{Conflict of Interests}

The authors declare that there is no conflict of interests regarding the publication of this paper.

\section{Acknowledgments}

The financial support of NSERC (Natural Sciences and Engineering Research Council of Canada), FQRNT (Fonds Québecois de la Recherche sur la Nature et les Technologies), MITACS Canada, Pratt \& Whitney Canada, and CETIM which provided the experimental results are gratefully acknowledged.

\section{References}

[1] W. J. Wang and P. D. McFadden, "Early detection of gear failure by vibration analysis i. calculation of the time-frequency distribution," Mechanical Systems and Signal Processing, vol. 7, no. 3, pp. 193-203, 1993. 
[2] W. J. Wang and P. D. McFadden, "Early detection of gear failure by vibration analysis-II. Interpretation of the time-frequency distribution using image processing techniques," Mechanical Systems and Signal Processing, vol. 7, no. 3, pp. 205-215, 1993.

[3] M. S. Safizadeh, A. A. Lakis, and M. Thomas, "Gear fault diagnosis using time-frequency methods," in Proceedings of the 20th Seminar on Machinery Vibration, pp. 7.19-7.29, Canadian Machinery Vibration Association, Québec, Canada, 2002.

[4] M. S. Safizadeh, A. A. Lakis, and M. Thomas, "Using shorttime fourier transform in machinery diagnosis," in Proceedings of the 4th WSEAS International Conference on Electronic, Signal Processing and Control (ESPOCO '05), pp. 494-200, Rio de Janeiro, Brazil, April 2005.

[5] Z. K. Peng and F. L. Chu, "Application of the wavelet transform in machine condition monitoring and fault diagnostics: a review with bibliography," Mechanical Systems and Signal Processing, vol. 18, no. 2, pp. 199-221, 2004.

[6] R. Yan, R. X. Gao, and X. Chen, "Wavelets for fault diagnosis of rotary machines: a review with applications," Signal Processing, vol. 96, pp. 1-15, 2014.

[7] N. Baydar and A. Ball, "Detection of gear deterioration under varying load conditions by using the instantaneous power spectrum," Mechanical Systems and Signal Processing, vol. 14, no. 6, pp. 907-921, 2000.

[8] N. Baydar, Q. Chen, A. Ball, and U. Kruger, "Detection of incipient tooth defect in helical gears using multivariate statistics," Mechanical Systems and Signal Processing, vol. 15, no. 2, pp. 303321, 2001.

[9] N. Baydar and A. Ball, "A comparative study of acoustic and vibration signals in detection of gear failures using Wigner-Ville distribution," Mechanical Systems and Signal Processing, vol. 15, no. 6, pp. 1091-1107, 2001.

[10] N. Baydar and A. Ball, "Detection of gear failures via vibration and acoustic signals using wavelet transform," Mechanical Systems and Signal Processing, vol. 17, no. 4, pp. 787-804, 2003.

[11] I. Yesilyurt, "The application of the conditional moments analysis to gearbox fault detection-a comparative study using the spectrogram and scalogram," NDT\&E International, vol. 37, no. 4, pp. 309-320, 2004.

[12] A. Parey and N. Tandon, Spur Gear Dynamic Models Including Defects: A Review, Sage, Thousand Oaks, Calif, USA, 2003.

[13] R. B. Randall, "A new method of modeling gear faults," ASME Journal of Mechanical Design, vol. 104, no. 2, pp. 259-267, 1982.

[14] P. D. Samuel and D. J. Pines, "A review of vibration-based techniques for helicopter transmission diagnostics," Journal of Sound and Vibration, vol. 282, no. 1-2, pp. 475-508, 2005.

[15] M. Lebold, K. McClintic, R. Campbell, C. Byington, and K. Maynard, "Review of vibration analysis methods for gearbox diagnostics and prognostics," in Proceedings of the 54th Meeting of the Society for Machinery Failure Prevention Technology, pp. 623-634, Virginia Beach, Va, USA, 2000.

[16] A. S. Sait and Y. I. Sharaf-Eldeen, "A review of gearbox condition monitoring based on vibration analysis techniques diagnostics and prognostics," in Rotating Machinery, Structural Health Monitoring, Shock and Vibration, Volume 5, Conference Proceedings of the Society for Experimental Mechanics Series, pp. 307-324, Springer, New York, NY, USA, 2011.

[17] P. Stevens, D. Hall, and E. Smith, "A multidisciplinary research approach to rotorcraft health and usage monitoring," in Proceedings of the 52nd Annual Forum of the American Helicopter Society, pp. 1732-1751, Washington, DC, USA, 1996.
[18] Z. Feng, M. J. Zuo, and F. Chu, "Application of regularization dimension to gear damage assessment," Mechanical Systems and Signal Processing, vol. 24, no. 4, pp. 1081-1098, 2010.

[19] S. Wu, M. J. Zuo, and A. Parey, "Simulation of spur gear dynamics and estimation of fault growth," Journal of Sound and Vibration, vol. 317, no. 3-5, pp. 608-624, 2008.

[20] R. Yan and R. X. Gao, "Approximate Entropy as a diagnostic tool for machine health monitoring," Mechanical Systems and Signal Processing, vol. 21, no. 2, pp. 824-839, 2007.

[21] W. J. Wang, J. Chen, X. K. Wu, and Z. T. Wu, “The application of some non-linear methods in rotating machinery fault diagnosis," Mechanical Systems and Signal Processing, vol. 15, no. 4, pp. 697-705, 2001.

[22] J. D. Jiang, J. Chen, and L. S. Qu, “The application of correlation dimension in gearbox condition monitoring," Journal of Sound and Vibration, vol. 223, no. 4, pp. 529-541, 1999.

[23] Y. He and X. Zhang, "Approximate entropy analysis of the acoustic emission from defects in rolling element bearings," Journal of Vibration and Acoustics, vol. 134, no. 6, Article ID 061012, 8 pages, 2012.

[24] L. Fu, Z.-Y. He, R.-K. Mai, and Q.-Q. Qian, "Application of approximate entropy to fault signal analysis in electric power system," Proceedings of the Chinese Society of Electrical Engineering, vol. 28, no. 28, pp. 68-73, 2008.

[25] Y. G. Xu, L. J. Li, and Z. J. He, "Approximate entropy and its applications in mechanical fault diagnosis," Information and Control, vol. 31, no. 6, pp. 547-551, 2002.

[26] R. Yan and R. X. Gao, "Complexity as a measure for machine health evaluation," IEEE Transactions on Instrumentation and Measurement, vol. 53, no. 4, pp. 1327-1334, 2004.

[27] J. Wang, L. Cui, H. Wang, and P. Chen, "Improved complexity based on time-frequency analysis in bearing quantitative diagnosis," Advances in Mechanical Engineering, vol. 2013, Article ID 258506, 11 pages, 2013.

[28] M. Kedadouche, T. Kidar, M. Thomas, and A. Tahan, "Combining EMD and Lempel-Ziv complexity for early detection of gear cracks," in Proceedings of the International Conference Surveillance 7, pp. 100-110, Chartres, France, October 2013.

[29] M. L. Dennis Wong, C. Liu, and K. Asoke Nandi, Classification of Ball Bearing Faults Using Entropic Measures, Surveillance 7, Chartres, France.

[30] L. Zhang, G. Xiong, H. Liu, H. Zou, and W. Guo, "Bearing fault diagnosis using multi-scale entropy and adaptive neuro-fuzzy inference," Expert Systems With Applications, vol. 37, pp. 6077$6085,2010$.

[31] J. S. Richman and J. R. Moorman, "Physiological time series analysis using approximate entropy and sample entropy," The American Journal of Physiology Heart and Circulatory Physiology, vol. 278, pp. 2039-2049, 2000.

[32] V. Tuzcu and S. Nas, "Sample entropy analysis of heart rhythm following cardiac transplantation," in Proceedings of the IEEE International Conference on Systems, Man and Cybernetics, pp. 198-202, IEEE, October 2005.

[33] X. Chen, S. C. Irene, and C. H. Ki, "Comparison of the use of approximate entropy and sample entropy: applications to neural respiratory signal," in Proceedings of the 27th Annual Conference on Engineering in Medicine and Biology, pp. 4212-4215, IEEE, Shanghai, China, September 2005.

[34] Y.-H. Sun, H.-L. Jou, and J.-C. Wu, "Auxiliary diagnosis method for lead-acid battery health based on sample entropy," Energy Conversion and Management, vol. 50, no. 9, pp. 2250-2256, 2009. 
[35] A. Widodo, M.-C. Shim, W. Caesarendra, and B.-S. Yang, "Intelligent prognostics for battery health monitoring based on sample entropy," Expert Systems with Applications, vol. 38, no. 9, pp. 11763-11769, 2011.

[36] A. Lempel and J. Ziv, "On the complexity of finite sequences," IEEE Transactions on Information Theory, vol. 22, no. 1, pp. 7581, 1976.

[37] M. El Badaoui, Contribution of vibratory diagnostic of gearbox by Cepstral analysis [Ph.D. thesis], Jean Monnet University, SaintÉtienne, France, 1999, (French).

[38] A. Pareya, M. El Badaoui, F. Guillet, and N. Tandon, "Dynamic modelling of spur gear pair and application of empirical mode decomposition-based statistical analysis for early detection of localized tooth defect," Journal of Sound and Vibration, vol. 294, no. 3, pp. 547-561, 2006. 

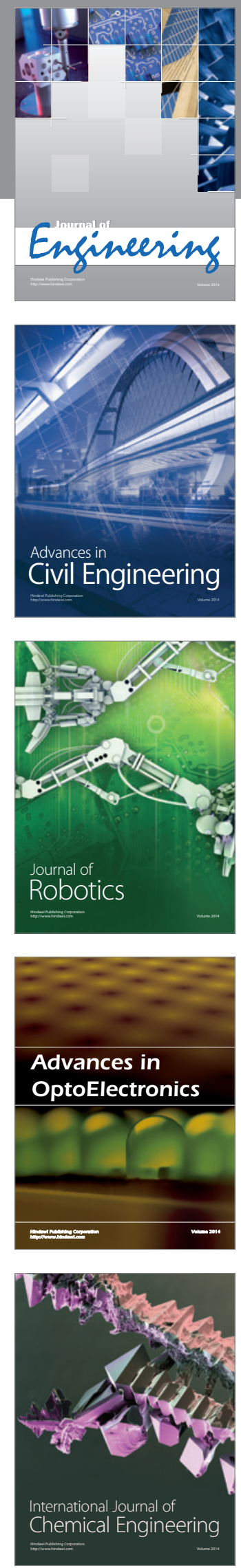

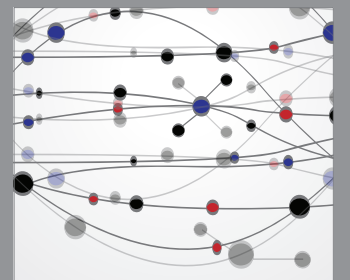

The Scientific World Journal
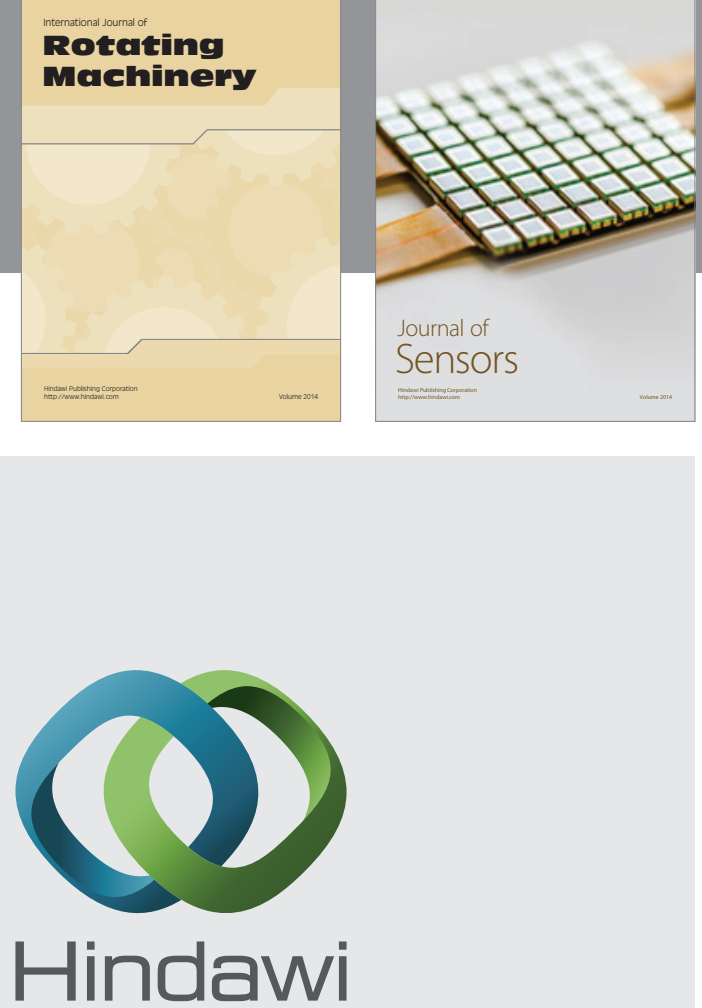

Submit your manuscripts at http://www.hindawi.com
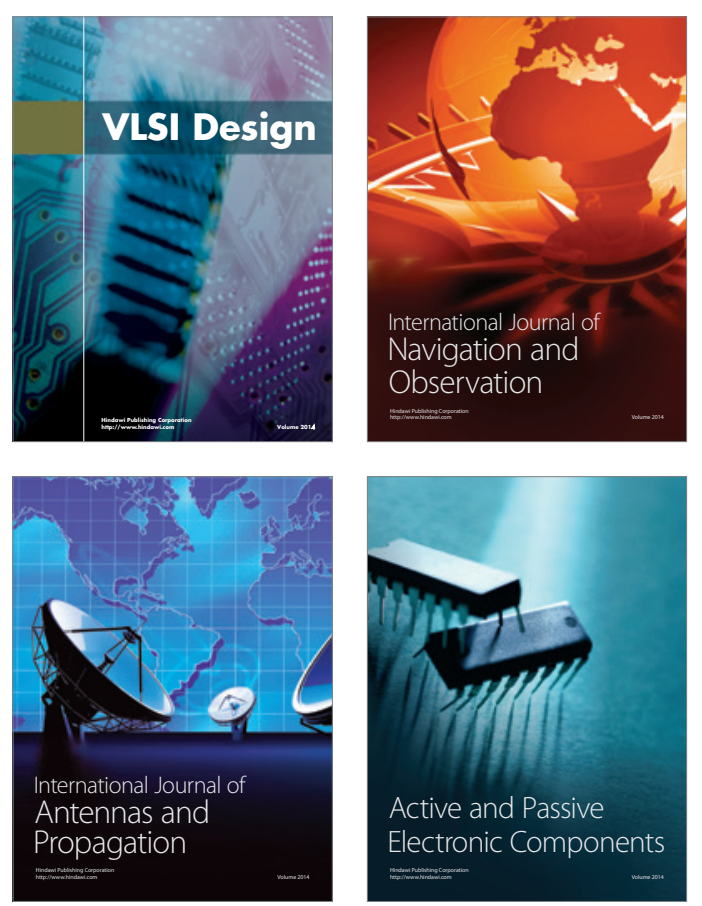
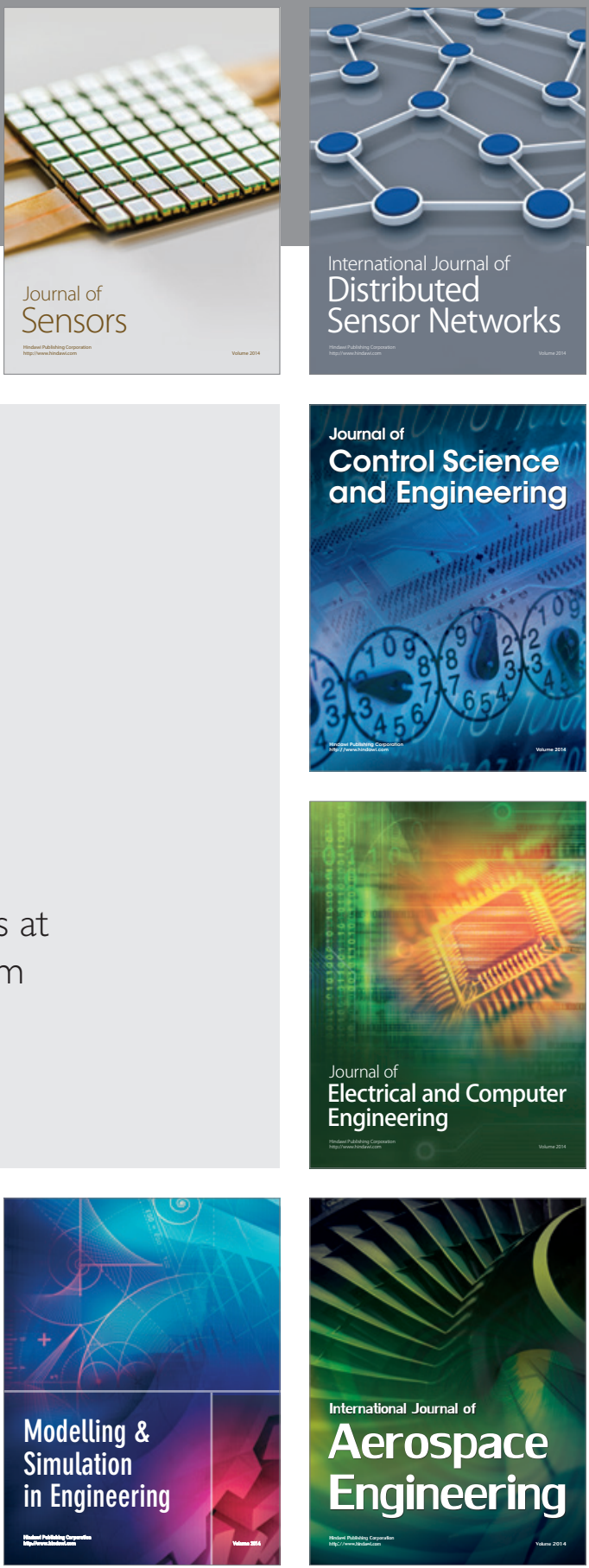

Journal of

Control Science

and Engineering
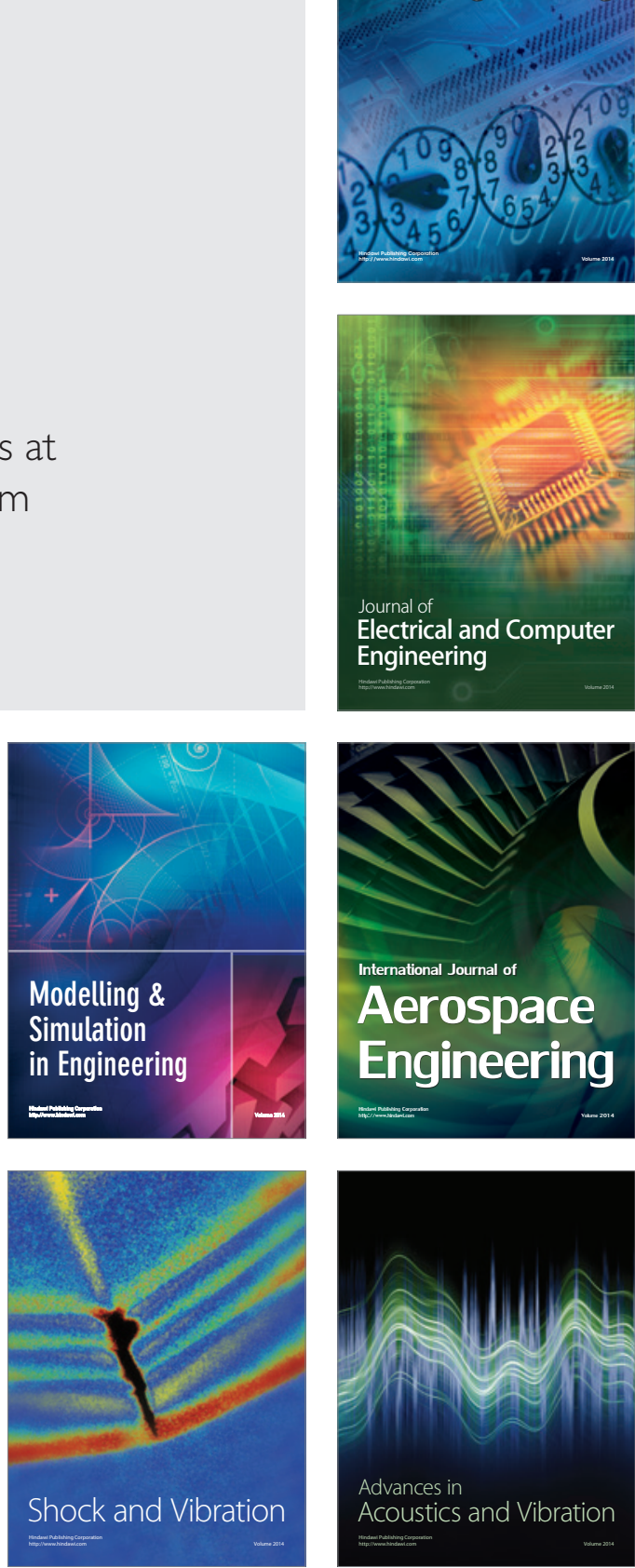